\title{
Increasing incidence of dementia in the oldest old: evidence and implications
}

\author{
Bryan D James* ${ }^{1 *}$ and Julie A Schneider ${ }^{2}$
}

\begin{abstract}
The oldest old are the fastest growing segment of the US population but accurate estimates of the incidence of dementia in this age group have been elusive. Corrada and colleagues present data on the 5 -year age-specific rates of dementia incidence in persons 90 years and older from The 90+ Study. Their findings show a continued exponential increase in dementia incidence after age 90 that mirrors the increase observed in persons aged 65 to 90, with a doubling every 5.5 years. This contrasts with previous smaller studies reporting a slowing of the increase in incidence after age 90. If confirmed, the continued increase, rather than a plateau, in the incidence of dementia in the oldest old has implications for proper healthcare planning. Strategies for prevention and treatment will require more information regarding risk factors and the etiopathogenesis of dementia in the oldest old.
\end{abstract}

The oldest old, persons living into the 10th and 11th decades of life, are the fastest growing segment of the population, with numbers expected to quadruple to over 8 million by 2050 [1]. Because advancing age is a major risk factor for dementia, this demographic shift may significantly increase the burden of dementia borne by our society. Indeed, the dementia incidence in persons aged 65 to 90 has been reported to double every 5 years [2]. However, few studies have had sufficient numbers of participants older than 90 years of age to provide accurate estimates in the oldest old and some studies have suggested a slowing of the incidence in the oldest old $[3,4]$. Further work is thus needed to determine the incidence of dementia in the oldest old. The present work not only has important implications for public health

*Correspondence: Bryan_James@rush.edu

'Rush Alzheimer's Disease Center, Room 1038, 600 South Paulina Street, Chicago, IL 60612, USA

Full list of author information is available at the end of the article planning, but is also relevant for studies of etiopathogenesis and strategies for prevention and treatment.

In a pivotal study addressing this question, Corrada and colleagues present data showing that the incidence of dementia continues to exponentially rise in the oldest old [5]. The authors studied 330 persons from The 90+ Study, a longitudinal cohort study of dementia in the oldest old. Participants were originally members of an epidemiological study of a retirement community in the 1980s [6]; 950 of the 1,150 persons at least 90 years old in 2003 were enrolled. Analyses for this study included only nondemented persons at baseline, as ascertained by inperson evaluation, who had at least one follow-up over 4 years. The authors found that the incident rate of dementia increased exponentially, from $12.7 \%$ per year for 90 to 94 year olds, to $21.2 \%$ per year for 95 to 99 year olds, to $40.7 \%$ per year for persons aged 100 years and older. The estimated doubling time for incidence rates was 5.5 years and is comparable with that observed for persons aged 65 to 90 .

The results of this study contrast with other studies that showed a slowing of dementia incidence after age 90 [7-13]. Importantly, the study has a number of strengths that strongly support the validity of the estimate. Having a much larger sample size, particularly for the 95 to 100 year olds and centenarians, allows for more stable estimates of incidence rates. Additionally, 6-month intervals between assessments and high follow-up rates (96\%) make it unlikely that loss to follow-up could erroneously result in an underestimate of incidence. There were some limitations, including the selective nature of the cohort, raising questions about selection bias and generalizability. The authors note that their study population predominantly female, white, with high education and socioeconomic status - is representative of individuals aged $90+$ in the US. Nonetheless, participants were originally recruited from one retirement community and agreed to participate in not one but two cohort studies, making selection bias still a potential issue. Epidemiologic studies with more racially and ethnically diverse cohorts will be important to confirm and extend these findings.

If confirmed, these data suggest a looming public health crisis. How can we prevent, delay, or treat dementia 
in the oldest old? To better address these questions, it will be important to determine the pathogenesis of dementia in the oldest old. Longitudinal clinicopathologic epidemiologic studies suggest that dementia in older persons is most commonly the result of mixed pathologies; most commonly Alzheimer's disease and vascular pathology [14-16]. While there are few pathologic data on the oldest old, a recent autopsy study in this group showed that mixed Alzheimer's disease and vascular pathology continues to increase with age [17]. Meanwhile, it has been suggested that the relationship between Alzheimer's disease pathology and dementia is attenuated in the oldest old [18]. This hypothesis may suggest a greater role for vascular factors or even suggest additional unrecognized pathologies. Further study of aging, dementia, and pathology in longitudinal community-based cohorts will be needed to address these important questions.

As the first major study to report that dementia incidence continues to rise exponentially in the oldest old, the present study has implications for how we conceive of the relationship between aging and dementia. It has been argued that a plateau in incidence in the oldest old indicates that dementia is not an 'aging-related' process - that is, an inevitable part of aging [19] - but rather an 'age-related' process, due to potentially preventable age-related risk factors $[4,12,13]$. Yet the distinction between these two concepts may be artificial. Indeed, the continued rise in incidence could result from the continued accumulation of age-related risk factors eventually surpassing the brain's compensatory facilities (that is, reserve capacity). In addition, even if some components of the pathologic process are inevitable, given the possible continued accumulation of Alzheimer's disease and mixed pathologies, it remains quite feasible that targeted strategies could prevent, delay, or treat at least part of the dementing process in the oldest old. After all, public health initiatives have dramatically pushed back the average age of death, the most inevitable consequence of aging.

\section{Competing interests}

JAS is a neuropathology consultant for AVID Radiopharmaceuticals, Inc. and is on the Alzheimer's Medical Advisory Board for GE Healthcare. The authors declare no other competing interests.

\section{Author details}

'Rush Alzheimer's Disease Center, 600 South Paulina Street, Room 1038 Chicago, IL 60612, USA. Department of Medicine, Rush University Medical Center, Chicago, IL, USA. ²Rush Alzheimer's Disease Center, 600 South Paulina Street, Suite 1022F, Chicago IL 60612, USA. Departments of Pathology
(Neuropathology) and Neurological Sciences, Rush University Medical Center, Chicago, IL, USA.

Published: 13 May 2010

\section{References}

1. US Census Bureau: Current Population Reports, Special Studies, P23-209, 65+ in the United States: 2005. Washington DC: Government Printing Office; 2005.

2. Jorm AF, Jolley D: The incidence of dementia: a meta-analysis. Neurology 1998, 51:728-733.

3. Gao S, Hendrie HC, Hall KS, Hui S: The relationships between age, sex, and the incidence of dementia and Alzheimer disease: a meta-analysis. Arch Gen Psychiatry 1998, 55:809-815.

4. Ritchie K, Kildea D: Is senile dementia 'age-related' or 'ageing-related'? Evidence from meta-analysis of dementia prevalence in the oldest old. Lancet 1995, 346:931-934.

5. Corrada MM, Brookmeyer R, Paganini-Hill A, Berlau D, Kawas CH: Dementia incidence continues to increase with age in the oldest old: the $90+$ study. Ann Neurol 2010, 67:114-121.

6. Paganini-Hill A, Ross RK, Henderson BE: Prevalence of chronic disease and health practices in a retirement community. J Chronic Dis 1986, 39:699-707.

7. Hagnell O, Ojesjo L, Rorsman B: Incidence of dementia in the Lundby Study. Neuroepidemiology 1992, 11(Suppl 1):61-66.

8. Fichter MM, Schroppel H, Meller I: Incidence of dementia in a Munich community sample of the oldest old. Eur Arch Psychiatry Clin Neurosci 1996, 246:320-328.

9. Canadian Study of Health and Aging Working Group: The incidence of dementia in Canada. Neurology 2000, 55:66-73.

10. Ruitenberg A, Ott A, van Swieten JC, Hofman A, Breteler MM: Incidence of dementia: does gender make a difference? Neurobiol Aging 2001, 22:575-580.

11. Edland SD, Rocca WA, Petersen RC, Cha RH, Kokmen E: Dementia and Alzheimer disease incidence rates do not vary by sex in Rochester, Minn. Arch Neurol 2002, 59:1589-1593.

12. Miech RA, Breitner JC, Zandi PP, Khachaturian AS, Anthony JC, Mayer L: Incidence of AD may decline in the early 90 s for men, later for women: the Cache County study. Neurology 2002, 58:209-218.

13. Hall CB, Verghese J, Sliwinski M, Chen Z, Katz M, Derby C, Lipton RB: Dementia incidence may increase more slowly after age 90: results from the Bronx Aging Study. Neurology 2005, 65:882-886.

14. Sonnen JA, Larson EB, Haneuse S, Woltjer R, Li G, Crane PK, Craft S, Montine TJ: Neuropathology in the adult changes in thought study: a review. J Alzheimers Dis 2009, 18:703-711.

15. Brayne C, Richardson K, Matthews FE, Fleming J, Hunter S, Xuereb JH, Paykel E, Mukaetova-Ladinska EB, Huppert FA, O'Sullivan A, Dening T: Neuropathological correlates of dementia in over-80-year-old brain donors from the population-based Cambridge city over-75s cohort (CC75C) study. J Alzheimers Dis 2009, 18:645-658.

16. Schneider JA, Arvanitakis Z, Bang W, Bennett DA: Mixed brain pathologies account for most dementia cases in community-dwelling older persons. Neurology 2007, 69:2197-2204

17. Jellinger KA, Attems J: Prevalence of dementia disorders in the oldest-old: an autopsy study. Acta Neuropathol 2010, 119:421-433.

18. Savva GM, Wharton SB, Ince PG, Forster G, Matthews FE, Brayne C: Age, neuropathology, and dementia. N Engl J Med 2009, 360:2302-2309.

19. Drachman DA: If we live long enough, will we all be demented? Neurology 1994, 44:1563-1565.

doi:10.1186/alzrt32

Cite this article as: James BD, Schneider JA: Increasing incidence of dementia in the oldest old: evidence and implications. Alzheimer's Research \& Therapy 2010, 2:9. 